\title{
DIGITAL SURVEY AND PARAMETRIC 3D MODELLING FOR THE VULNERABILITY ASSESSMENT OF MASONRY HERITAGE: THE BASILICA OF SAN DOMENICO IN SIENA, ITALY
}

\author{
ANGELO MASSAFRA, CARLO COSTANTINO, DAVIDE PRATI, \\ GIORGIA PREDARI \& RICCARDO GULLI \\ Department of Architecture, Alma Mater Studiorum, University of Bologna, Italy
}

\begin{abstract}
This research focuses on the combined use of digital survey tools and parametric 3D modelling procedures for supporting the vulnerability analysis of masonry heritage. The behaviour of masonry structures is strongly related to the geometrical properties of macro-elements, their connections, and material characteristics. The collapse of these buildings occurs mainly due to the loss of equilibrium of the macro-elements than the breaking of the materials. Moreover, the numerical computing of structural element stresses requires an in-depth knowledge of material properties that could be reached mainly through destructive tests, which have high costs and could damage the architectural figurativeness of protected buildings. Besides, many tests are required because of the high variability of mechanical properties within an ancient masonry building due to the various transformations and construction techniques that usually characterise its construction history. A quick and non-destructive approach is proposed as a preliminary support tool. It is based on the observation of high-resolution unmanned aerial vehicles (UAV) orthophotos combined with terrestrial laser scanning (TLS) point cloud geometric information analysis through visual programming generative algorithms. The case study is the Basilica of San Domenico, one of the most important churches in Siena, Italy, built in the 13th century, completed in the 15th century, and repeatedly modified until the beginning of the 20th century. The Basilica has construction features (the large volume without side chapels and high slenderness) that make it particularly vulnerable to horizontal actions. These inherent vulnerabilities were joined by the effects of an earthquake in 1798, whose damages were partially remedied. The workflow application allowed highlighting the hypothetical displacements that the transept and the bell tower have undergone over time, improving their behaviour comprehension. It also helped to point out the critical issues of the whole building, supporting the vulnerability analyses based on the macro-elements and orienting the intervention hypothesis for its preservation.

Keywords: cultural heritage, Basilica of San Domenico in Siena, masonry structures, terrestrial laser scanning, UAV photogrammetry, generative algorithms, parametric 3D modelling, structural systems reverse engineering, displacement analysis.
\end{abstract}

\section{INTRODUCTION}

The importance of preserving masonry heritage cannot be disregarded since most of the historical buildings in Europe were built with this construction technique. Effective ways of retaining these architectures are needed, based on appropriate knowledge of their structural systems. Minimally invasive approaches are required, especially on protected buildings, to carry out an aware conservation process without damaging them.

In general, to evaluate the seismic risk assessment, a simplified qualitative procedure is implemented to have a first overview of the main vulnerability of damaged heritage buildings after an earthquake. This evaluation, specific for protected buildings, is valid as a preliminary analysis but not helpful in designing targeted interventions for seismic improvement that require more complex quantitative assessment [1]. 
In the case of churches, the simplified procedure is reduced to defining a single parameter $\mathrm{i}_{\mathrm{v}}$ (vulnerability index) [2]. Great importance is given to the assessments of out-of-plane mechanisms (1st mode) with linear kinematic analysis, which is typically done by decomposing the structure into macro-elements. In fact, studies related to earthquake damages undergone by churches, carried out during the last decades' main Italian seismic events, have highlighted how their seismic behaviour is characterised by an autonomous structural response of the macro-elements rather than of the church as a whole structure. This happens because, as for most historic masonry buildings, disconnections of the construction elements occur and do not guarantee structural continuity, causing the earthquake action to further increase these disconnections.

Although these qualitative assessments help deepen the analyses of the construction and the cracking framework of masonry buildings, which are still the most reliable path towards evaluating numerical assessments, they strongly depend on the technician's judgment validating the interpretation of the activated kinematic chains.

Therefore, it has been decided to exploit the point cloud data obtained with on-site complementary terrestrial laser scanning (TLS) and aerial photogrammetry (UAV) techniques to support and make more effective evaluations and increase the objectivity value of the qualitative analyses. In particular, the use of generative algorithms already developed to analyse other construction elements [3], [4] has allowed deepening the automation of the analysis of the out-of-plane of the transept façades and bell tower of the Basilica of San Domenico in Siena, Italy. Similar studies that combine digital survey techniques and out-ofplane analysis are not widespread in scientific literature and often require a complex and dedicated survey campaign [5], [6].

\section{THE CASE STUDY}

The presented case study is the Basilica of San Domenico, a masonry church built in the 13th century on Camporegio Hill in Siena, Italy, near the medieval fountain of Fontebranda. On the one hand, the prime location ensures the church a landmark role over the city along with the Palazzo Pubblico tower and the Cathedral bell tower. On the other hand, it has been the leading cause of many structural issues since its construction.

Although the Dominican Order settled in Siena in 1221, the documentary sources indicate that San Domenico's nave construction began only around 1246, and it was completed around 1300. Some documentary sources suggest that the construction incorporated the ancient church of San Gregorio, but this has never been confirmed [7], [8]. The first expansion of the Basilica began even before the nave was completed and concerned the Beato Ambrogio Chapel's construction. The location of the chapel, demolished in 1479, was at the corner between the nave, the current bell tower, and the transept [8]. The construction of the Lower Church between 1306 and 1352 turned out to be one of the most critical phases for the contemporary church shape; in fact, it would have been employed as a foundation for the future transept. However, the plague interrupted the public and private donations for the construction progress, and therefore it was suspended for over thirty years. Indeed, the transept was completed only between 1380 and 1480 through many difficulties and interruptions, using the chapels owner families' funds [9].

The bell tower is documented since 1321, but the current construction is a later work dating between 1489 and 1517 when the statue of St. Caterina from Siena was placed on the top spire. Later on, the spire and the statue were removed due to the excessive danger caused by lightning bolts.

Between the 16th and 18th centuries, many interventions were carried out: the reconstruction of the roof, changes on the openings, altars addition and other Baroque 
decorations in the nave, the consolidation of the masonry. In 1798, the church was severely damaged by an earthquake to the point that its demolition and reconstruction were considered. Only after a lot of debate, the Sienese population opted for its restoration.

Finally, extensive renovation work was carried out during the 20th century to uncovered as far as possible the medieval frescos and restore the original natural illumination coming from the large arched windows.

\section{THE INVESTIGATION PROTOCOL WORKFLOW}

The research allowed defining a procedure to systematise the assessment of masonry structures' behaviour in terms of displacement and deformations over time (Fig. 1). This research protocol includes an on-site instrumental acquisition phase using UAV photogrammetry and TLS survey techniques, followed by the digital rendering of the acquired data, extracting high-resolution orthophotos and generating 3D point clouds. The orthophotos are carefully observed to identify the building conservation state, cracks, and damages. Consequently, the vulnerabilities and the associated damage mechanism (e.g., the overturning of the façade or diagonal cracks on the transversal wall) can be evaluated starting from the crack patterns. At the same time, parametric models from the TLS point cloud permit highlighting the hypothetical displacements that macro-elements have undergone during the lifespan.

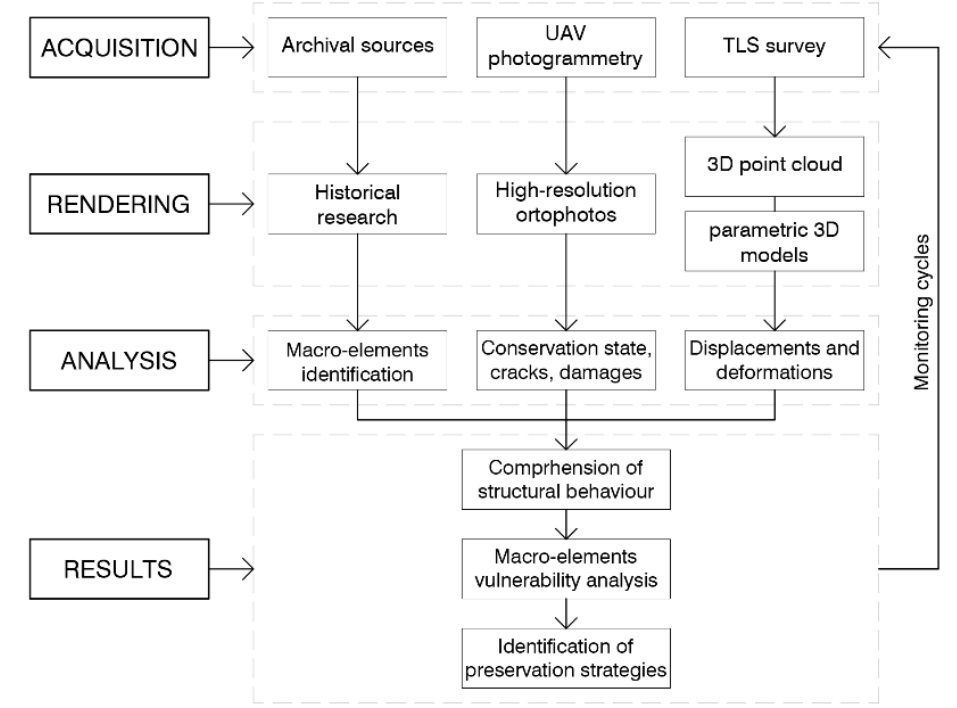

Figure 1: Investigation protocol workflow. (Source: Authors' graphic elaboration.)

These methodologies allow examining in detail building portions that would have been impossible to investigate with traditional methods because of their heights (up to about $40 \mathrm{~m}$ ), the obstacles hiding them from the ground, or the occlusion generated by other buildings or vegetation. By combining these different analyses with historical data, it is possible to understand the investigated buildings' effective structural behaviour. The protocol allows to precisely recognise the crack pattern and connect it to the most probable collapse kinematics of masonry elements, which is the key for numerically assessing the vulnerability macro-elements analysis, orienting the intervention hypothesis for the building preservation. 
Finally, future repetitions of the whole protocol would permit efficient monitoring cycles regarding the displacements and the conservation state of the buildings over time.

\subsection{The TLS and UAV photogrammetric survey}

The TLS and UAV photogrammetric survey campaign took five days. It was conducted employing a FARO CAM2 FOCUS $3 \mathrm{D}^{\circledR}$ and a DJI Mavic Mini, an open category UAV of C0-class identification label (MTOM $<250 \mathrm{~g}$ ), using a targetless approach on both types of equipment. The photogrammetry needed, at least a $50 \%$ overlapping of the pictures with a proper resolution, to ensure an ideal outcome. These images were elaborated in Agisoft Metashape $^{\circledR}$ 1.6.5 to make the photogrammetric 3D model and orthophotos of both the external and internal elevation (Fig. 2). Overall, the total airtime was about $8 \mathrm{~h}$, allowing the shooting of 708 pictures.

On the other hand, for the entire TLS survey of the building (Fig. 3), it was necessary to shoot 130 scans with a medium resolution of $7.67 \mathrm{~mm} / 10 \mathrm{~m}$ and a quality filter of $3 \times$. With a medium overlapping between the scans of $36 \%$, despite the building complexity and size, it was possible to achieve an extremely accurate alignment, with an average $1.5 \mathrm{~mm}$ standard deviation between corresponding points and a maximum $2.6 \mathrm{~mm}$ deviation.

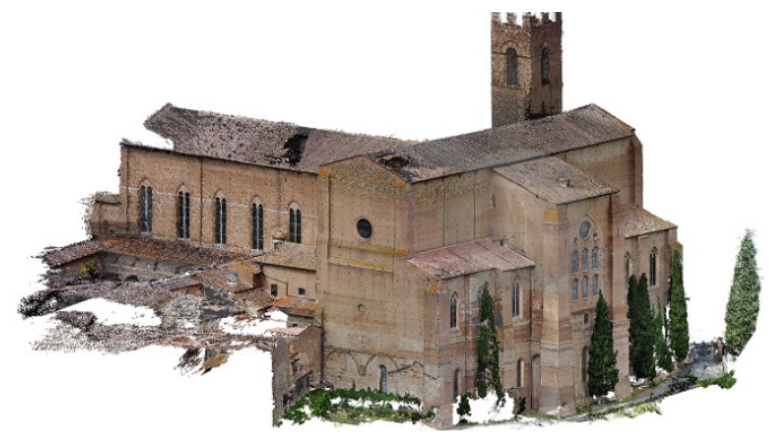

Figure 2: The photogrammetric mesh model of the exterior of the Basilica of San Domenico in Siena. (Source: Authors' graphic elaboration.)

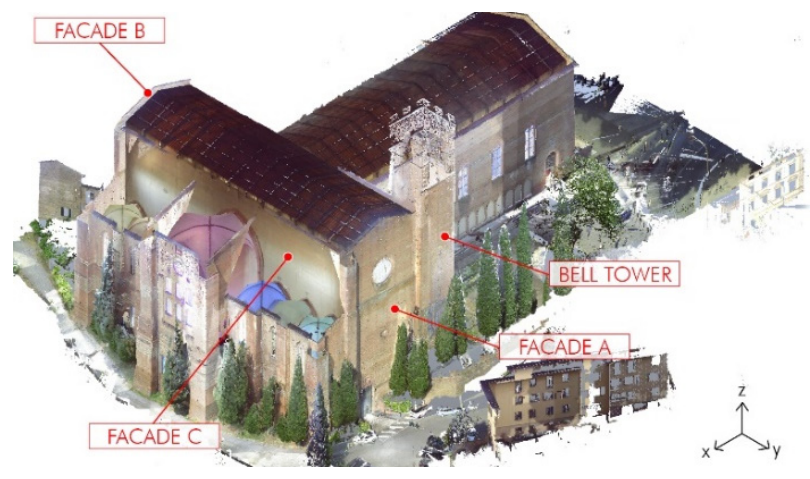

Figure 3: TLS point cloud of the Basilica of San Domenico in Siena: identification of the investigated masonry walls and the reference system. (Source: Authors' graphic elaboration.) 
The remarkable height of the Basilica, which reaches $42.85 \mathrm{~m}$ in the transept and $48.25 \mathrm{~m}$ in the bell tower, the difficulties in surveying near the steep slopes of the hillside, and in getting precise information of the upper part of the inner transept were the main challenges of the survey campaign.

\subsection{High-resolution orthophotos and crack patterns analysis}

The use of high-resolution orthophotos, made from UAV photogrammetry techniques, consists of a valuable and effective way to analyse the masonry building crack patterns and compare the cracks present on the inside wall with the outside ones. Having the exact correspondence between the external and the internal cracks allows to accurately value their locations, their width, and if they concern the entire thickness of walls.

The orthophotos of the Basilica of San Domenico have been used to divide the cracks according to their width into three categories: small cracks $(<2 \mathrm{~mm})$; medium cracks $(2 \mathrm{~mm}$ $\div 1 \mathrm{~cm}$ ); dangerous cracks $(>1 \mathrm{~cm})$. Simultaneously, those walls characterised by mortar absence were detected, and those areas where the previous cracks have been reintegrated. Based on all these considerations, the mechanisms associated with their corresponding crack patterns have been defined as follow: (1) Out-of-plane flexures; (2) Diagonal shear cracks; (3) Anchor-rod punching; (4) Do-not-restrained arch trusts; (5) Crushing cracks; (6) Cracks related to different construction phases; and (7) Foundation subsidence.

The crack patterns analysis identified vertical cracks due to out-of-plane overturning at the corners of the transept heads (façades A and B). Those on façade B are particularly visible, as they reach $5-6 \mathrm{~cm}$ in width. This mechanism is also confirmed inside the church by similar vertical cracks, albeit with a smaller width.

Furthermore, in the B façade, there are numerous cracks (Fig. 4), which follow a subvertical direction in the upper part of the elevation and become diagonal near the base. Other minor damages are attributable to the roof trusses' concentrated loads and the masonry's poor quality and condition in correspondence with the tympanum. The crack pattern in the inner transept surface is complex and referable to multiple factors as the out-of-plane overturning of the chapels, the instability of the corner section of the wall, the shear cracks, the midheight-out-of-plane overturning, the do-not-restrained arch thrust of the choir. Furthermore, it cannot be excluded that these mechanisms are also caused by local foundation subsidence.

\subsection{The model generation procedure}

The assumption behind the algorithmic 3D model generation procedure is to take advantage of the large amounts and accuracy of spatial information provided by TLS devices, such as point clouds. These data allow a highly detailed analysis of the masonry elements and, therefore, the elaboration of specific and comparative information on their static behaviour and their state of preservation. The usual modelling methodologies, such as vectorisation of orthophotos or 3D modelling using CAD or BIM software, heavily depend on the operator's choices and are affected by low repeatability and accuracy. For these reasons, it was decided to transform the point cloud into 3D models using Grasshopper ${ }^{\circledR}$ generative algorithms. These tools, which are continuously updated, provide advantages over manual modelling methods, both in the speed of execution and the elimination of inaccuracies. 


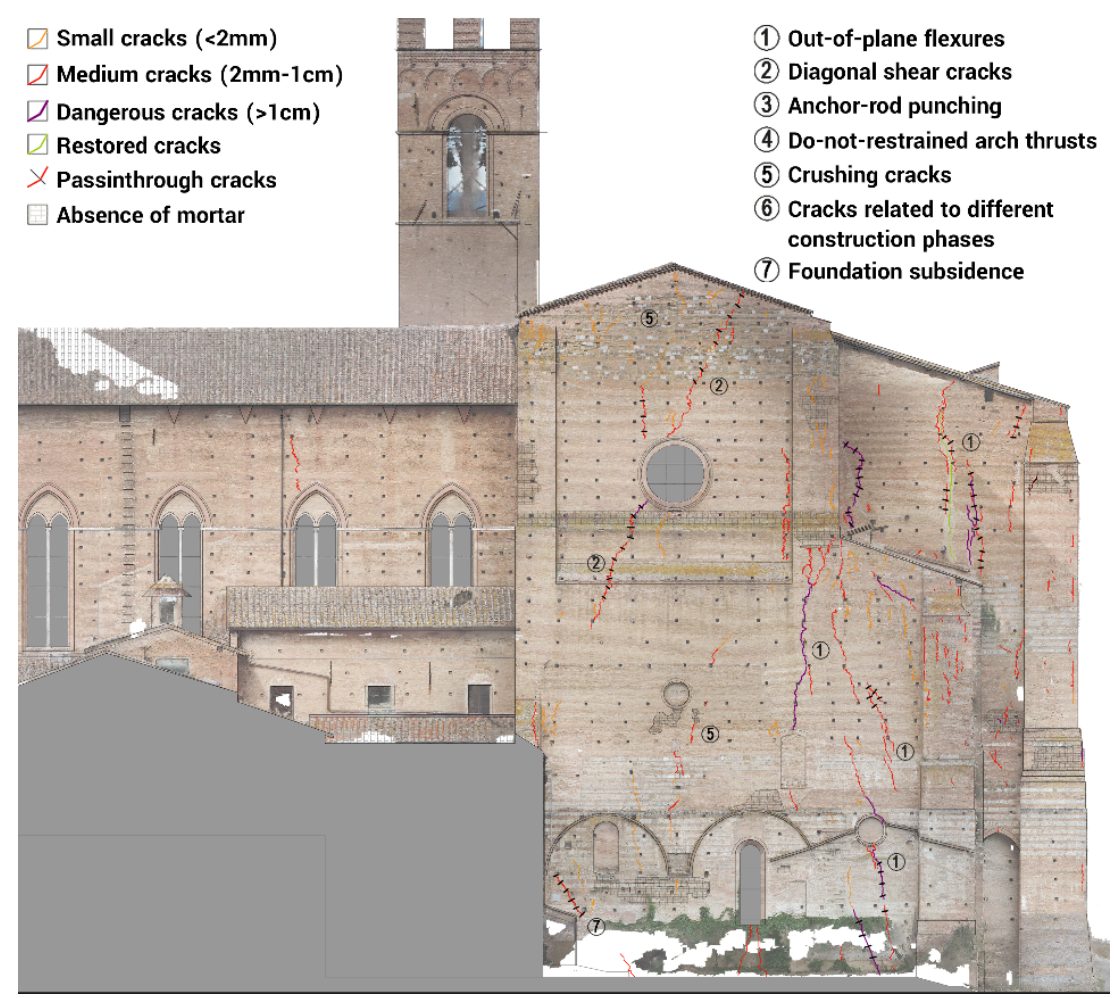

Figure 4: Façade B of the Basilica. Orthophoto highlighting the crack patterns and their interpretation and analysis. (Source: Authors' graphic elaboration.)

\subsubsection{Parametric 3D modelling}

The parametric modelling workflow was applied to each façade identified in Fig. 2, considering the reference system shown in the same figure. The $\mathrm{x}$-axis is parallel to the nave with the positive direction towards the valley, the y-axis is parallel to the transept with the positive direction towards the bell tower, the z-axis is zenith with the positive direction towards the sky.

The segmented point cloud of the façade, which is also called the "Basic 3D Model" as it represents the structure's current condition, was transformed into the "Ideal 3D Model" through generative algorithms. This model represents the wall's hypothetical undeformed configuration, excluding the out-of-plane deformations that have presumably occurred over time. In particular, the Ideal 3D Model coincides with the "average vertical plane" of the façade, in other words, the plane that represents the ideal condition of a perfectly flat and vertical façade.

The operations for generating the Ideal Plane are illustrated in Fig. 5. Firstly, the point cloud of the façade was segmented and cleaned up in Geomagic Control ${ }^{\circledR}$; it was divided in the inner and outer surface, horizontal bands of points were selected, exported into Rhino ${ }^{\circledR}$, and linked to the Grasshopper ${ }^{\circledR}$ algorithm (Fig. 5). The algorithm vectorised the horizontal cross-sections of the wall (in this case, it was chosen to consider a section-curve every $2 \mathrm{~m}$ of height). Then the algorithm selected the portion of the curves that best represented the planar condition of the façade, removing pilasters, buttresses, protrusions, recesses, etcetera. 
If the façade had different main planes, which means that it presents different section thicknesses, only the curves relating to the lowest plane of the façade were selected. These curves were divided into a certain number of points (e.g., 100 points) and, for each group of points, the "linear regression line" was calculated. This line is the one-degree polynomial curve, lying in the horizontal plane, that best interpolates the points group. A similar procedure was carried out for calculating the "vertical linear regression plane", which is the plane that best interpolates the best-fit lines, forced to be perfectly vertical (Fig. 5).

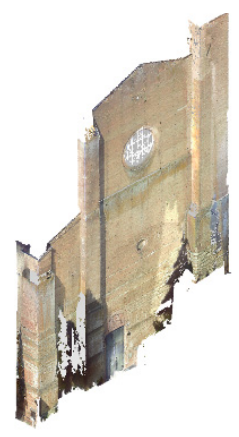

(a)

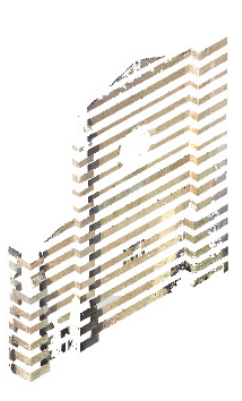

(b)

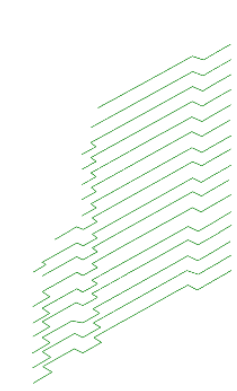

(c)

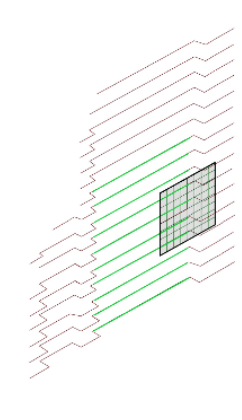

(d)

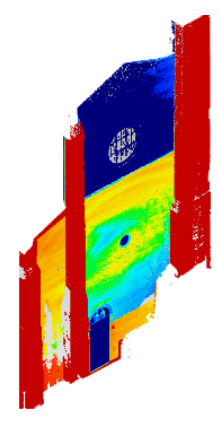

(e)

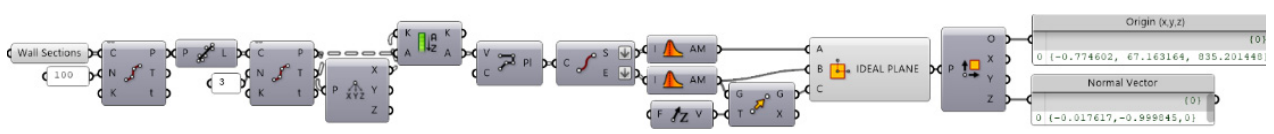

Figure 5: The model generation procedure and grasshopper algorithm. (a) TLS point cloud; (b) Point cloud segmentation; (c) Vectorised cross-sections; (d) Ideal Plane; and (e) TLS point cloud-Ideal Plane comparison. (Source: Authors' graphic elaboration.)

This plane is the Ideal Plane that represents the ideal condition of the wall. It is important to note that this plane is not properly representative of the façade's condition at the time of its original construction since it is often difficult to find an absolute reference to attribute the original deformation state of the façade, especially in such complex buildings. However, it consists of a useful approximation to evaluate the façade areas that have lost flatness and could be considered a relative reference for assessing its out-of-plane deformation state. It permits knowing how much a point of the cloud deviates from the mean plane and identifying areas of the façade that show typical deviation values.

\subsubsection{D models and point cloud comparison}

Once the Ideal 3D Model definition was reached, a comparative analysis was performed using Geomagic Control $^{\circledR}$ to evaluate the deviations between the Ideal 3D Model (the ideal plane) and the Basic 3D Model (the TLS point cloud). For all the investigated masonry elements, the minimum and maximum reference deviation threshold were manually set in a chromatic scale to better indicate the extent of the movements that occurred between the surveyed configuration and the ideal one. The graphic representation through the most appropriate chromatic scale clarifies the entities of the displacements. It is possible to select any position on each portion of the masonry to highlight the local deviations' numerical value using the graphical representation (Figs 6-9). This representation provides qualitative and 
quantitative information and facilitates an accurate and widespread understanding of all the displacement and deformations that each wall has undergone.

Comparisons can be made in a 3D space and on 2D projection planes user-selected according to the deviation types that need to be highlighted. The software also allows for the precise quantification of deviations using control points. With the "annotation" tool, it was possible to report the exact value of the distances measured between the two compared objects' corresponding points and their subdivision along the $\mathrm{x}, \mathrm{y}$, and $\mathrm{z}$ axes.

\section{RESULTS}

The chromatic scale highlights deviation trends that could describe the typical kinematics that ancient masonry structures usually manifest and the interaction between the macroelements. The integration of the proposed displacement analysis with the usual crack patterns analysis, supported by the historical research and the in-situ inspections, allowed formulating hypotheses on the church's behaviour, producing interesting and coherent results.

\subsection{Façade A}

Façade A, adjacent to the bell tower, presents thickness change in elevation. Therefore, the analysis was performed firstly for the lower part, evaluating its deviations from the façade's mean plane model. The upper part was analysed with reference to the lower part's ideal plane, which was shifted in correspondence to the upper part's barycenter. More specifically, the upper part's mean plane is calculated first in a similar way to that of the lower part of the façade. Then the lower mean plane is translated to the origin of the upper mean plane. This translated plane is the Ideal 3D Model of the upper part of the façade.

The analyses show that the lower portion of the façade's exterior surface exhibits a relative offset between its base and top of about $9 \mathrm{~cm}$. The top is projecting outward from the building in the positive y-direction. The upper portion of the façade follows the same trend as the lower part. Moreover, it is rotated with respect to its mean xy-plane so that the side adjacent to the bell tower is projected towards the outside of the building (Fig. 6).

It is important to note that this rotation does not mean that the façade left side is rotated towards the inside of the building. More likely, this means that there is a delta deviation from the mean plane equal to about $9 \mathrm{~cm}$ caused by the interaction with the bell tower movement. The combined interpretation of both façade analyses suggests a simple tilting of the façade with some interference with the bell tower's movements (Fig. 7).

\subsection{Bell tower}

The bell tower's analysis was carried out on its two main elevations facing the outside of the building. As far as the tower elevation adjacent to façade A is concerned, the analyses show the displacements' compatibility since the bell tower also presents a relative displacement between its base and its top equal to about $7 \mathrm{~cm}$, with the top part projected towards the outside (similar to façade A value).

Observing the other elevation, parallel to the y-axis, the deviation between the top and the base is even more marked, equal to about $19 \mathrm{~cm}$ (Fig. 7). Therefore, it is possible to hypothesise a tilting of the bell tower in the direction towards the outside of the building. The top is about $7 \mathrm{~cm}$ displaced from the base in the positive $y$-direction and about $19 \mathrm{~cm}$ in the negative $x$-direction. Moreover, observing the façade's cracking pattern, a vertical lesion is evident at the border between façade A and the bell tower, which shows a separation between the two macro-elements. 

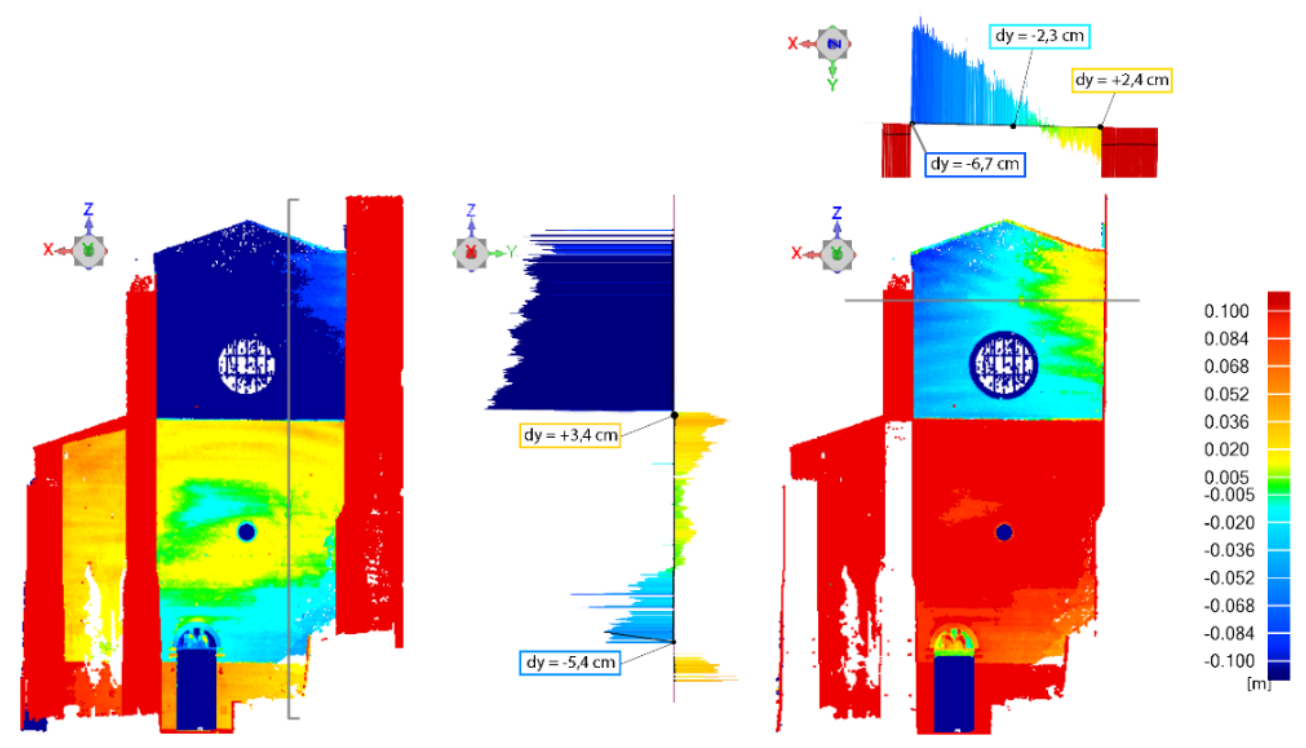

Figure 6: Displacement analysis of the façade A. The green points lie on the mean plane of the façade. The red points deviate from the mean plane in positive y-direction towards the outside of the building, the blue ones in the negative y-direction towards the inside of the building. The deviation values are represented on a scale 100 times larger to allow greater readability in the sections. (Source: Authors' graphic elaboration.)
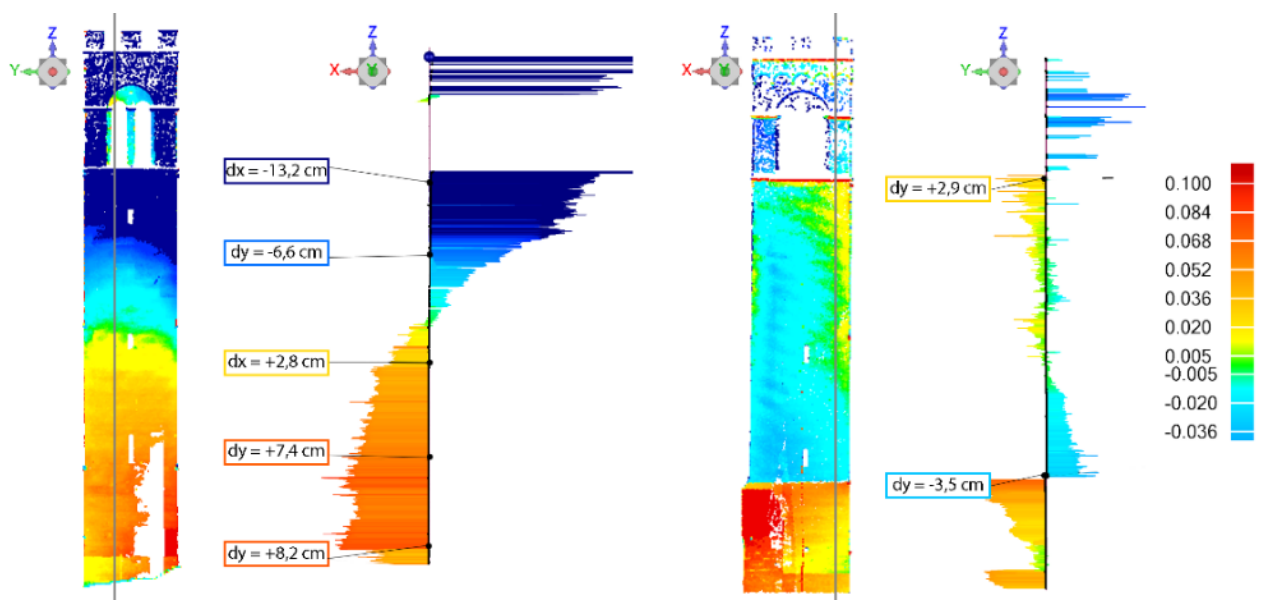

Figure 7: Displacement analysis of the bell tower. On the left, the red points deviate from the mean plane in the positive $\mathrm{x}$-direction towards the inside of the building, the blue points in the negative $\mathrm{x}$-direction towards the outside of the building. On the right, the red points deviate from the mean plane in the positive y-direction towards the outside of the building, the blue points in the negative y-direction towards the inside of the building. (Source: Authors' graphic elaboration.) 


\subsection{Façade B}

Façade B, which is on the opposite side of the transept with respect to façade A, presents the same thickness change in elevation. Therefore, the analysis is carried out following the same methods illustrated for façade A.

The analyses show that the lower part of the façade is substantially vertical. Only the right top seems to move slightly towards the outside of the building. By going deep in the 2D comparisons, a vertical overhang of about $10 \mathrm{~cm}$ can be highlighted, and a horizontal rotation with a delta of deviation between the downward side and the symmetrical one of about $5 \mathrm{~cm}$.

Also, in this case, the upper portion of the façade follows the same trend as the lower part and is rotated in the xy-plane with respect to its mean plane so that the side adjacent to the façade $\mathrm{C}$ is projected towards the outside of the building. The façade's left side seems rotated towards the inside of the building, but this analysis means that the overall façade delta of deviation from the mean plane is about $10 \mathrm{~cm}$ (Fig. 8).

The combined interpretation of the two parts of the façade suggests a combined tilting of the façade with interference with façade $\mathrm{C}$ movements (Fig. 9). The combined interpretation of the façade's two parts indicates a relationship between the deformations and the vertical lesion on the downstream side of the transept, adjacent to the cloister side façade. This lesion shows a detachment of the façade in its upper part, resulting in the façade's overturning.

\subsection{Façade C}

Since it was not possible to survey the façade $\mathrm{C}$ accurately, due to the high hill slope and the presence of a tree curtain, the internal surface was used for the analysis.
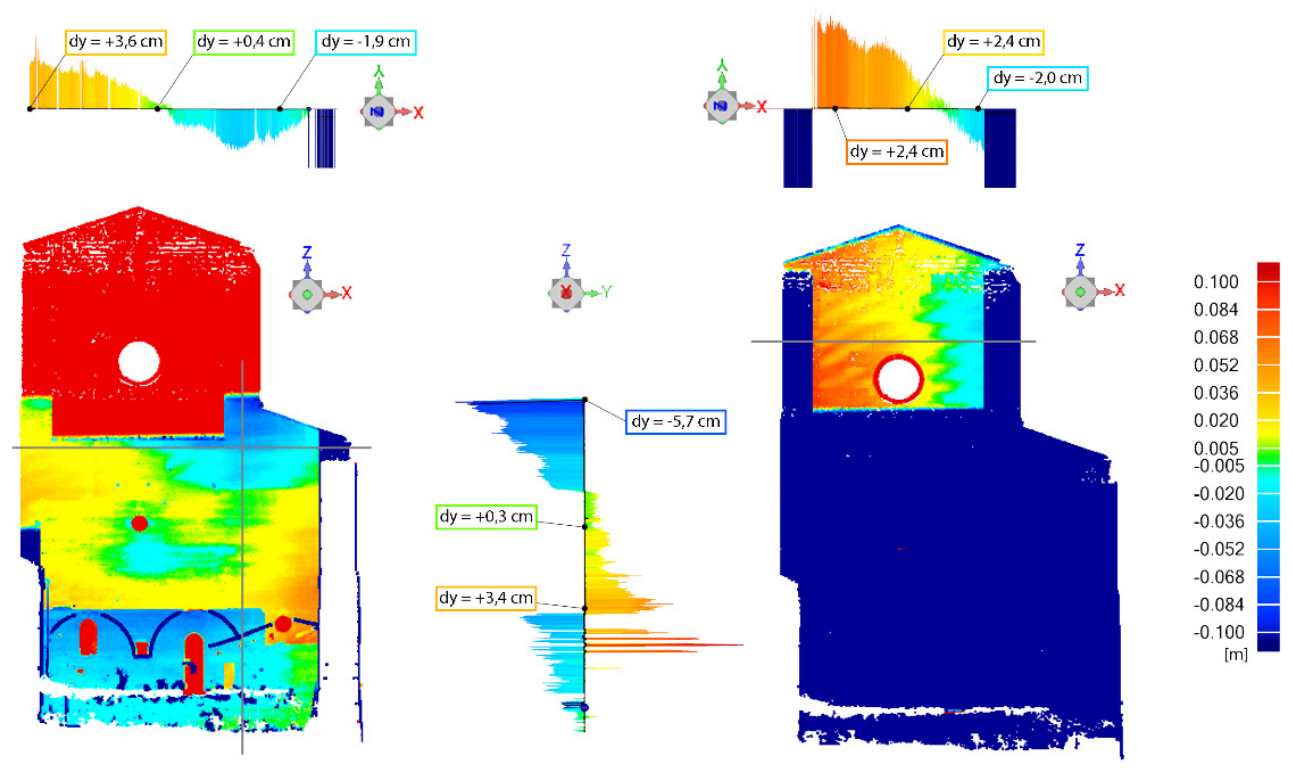

Figure 8: Displacement analysis of façade B. The red points deviate from the mean plane in the positive y-direction towards the inside of the building, the blue points in the negative y-direction towards the outside of the building. (Source: Authors' graphic elaboration.) 

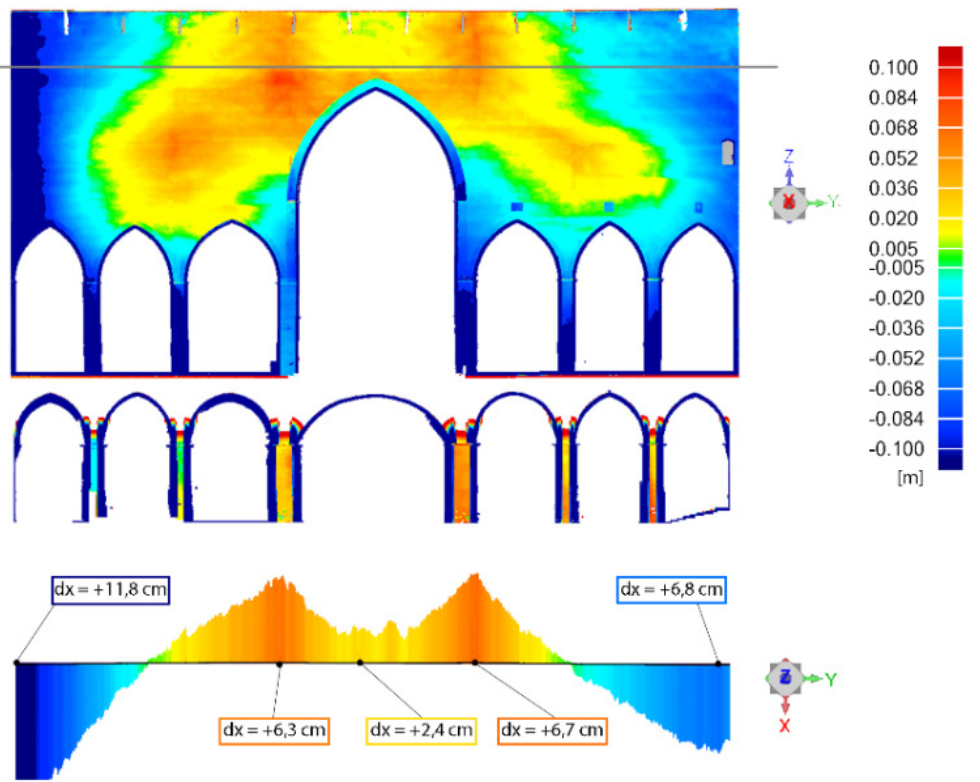

Figure 9: Displacement analysis of façade B. The red points deviate from the mean plane in the negative $\mathrm{x}$-direction towards the inside of the building, the blue points in the positive x-direction towards the outside of the building. (Source: Authors' graphic elaboration.)

From a qualitative point of view, the deformations of this part of the façade show how the lateral zones are offset from the mean façade plane towards the outside of the building, while the central zones are offset towards the inside of the building. Unlike all other façades, there is no particular offset in the vertical direction (Fig. 9).

Analysing the 2D comparison, the maximum deviation between the lateral and the central parts is equal to about $18 \mathrm{~cm}$. Moreover, the transition from the blue-coloured points (on the side) to the yellow-coloured ones (on the centre) occurs according to the so-called "deformation isolines" with about $45^{\circ}$ inclination. This supports the hypothesis of activation of a kinematic chain of combined overturning on both transept sides, with the interference of the deformations of façades A and B.

\section{CONCLUSION}

This research proposes a quick and non-destructive approach as a preliminary tool for supporting the vulnerability analysis of masonry heritage, based on the observation of the crack pattern through high-resolution UAV orthophotos combined with the analysis of TLS point cloud geometric information through visual programming generative algorithms.

The method could contribute to developing a more accurate and objective macroelements analysis that, even if it strongly depends on the subjectiveness of the technician's judgment, is the most reliable path towards evaluating numerical assessments for masonry buildings. In fact, many seismic events that occurred in the last decades in Italy demonstrated that the collapse of masonry structures occurs mainly due to the loss of equilibrium of the macro-elements than the breaking of the materials. 
The workflow was applied to the Basilica of San Domenico's case study in Siena, Italy, a 13th-century church with many construction features that make it particularly vulnerable to horizontal actions. The combined interpretation of the parametric 3D results with the analysis of the cracking framework confirmed the activation of some prevailing kinematic chains: a simple overturning of both façades $\mathrm{A}$ and $\mathrm{B}$ towards the outside of the building. This overturning probably led to the formation of the vertical lesion between façade $\mathrm{A}$ and the bell tower. Façade B presents a compound overturning with the detachment of the masonry wedge placed adjacent to façade $\mathrm{C}$. The presence of a marked vertical lesion between the two wall curtains also suggests that façade $\mathrm{B}$ has detached from façade $\mathrm{C}$, highlighting a simple overturning pattern. Moreover, façade $\mathrm{C}$ presents a compound overturn on both masonry wedges on the sides, highlighting relationships with façades $\mathrm{A}$ and $\mathrm{B}$.

The protocol's application allowed highlighting the hypothetical displacements that the transept and the bell tower have undergone over time, improving the comprehension of their behaviour and the critical issues of the whole building. It also supported the vulnerability analyses based on the macro-elements and oriented the intervention hypothesis for church preservation.

\section{ACKNOWLEDGEMENT}

This research is part of a series of activities jointly carried out between the Department of Architecture research group and the Soprintendenza Archeologia Belle Arti e Paesaggio per le province di Siena, Grosseto e Arezzo, which is currently underway.

\section{REFERENCES}

[1] Direttiva del Presidente del Consiglio dei Ministri 9 febbraio 2011. Valutazione e riduzione del rischio sismico del patrimonio culturale con riferimento alle Norme tecniche per le costruzioni di cui al D.M. 14/01/2008, 2011.

[2] Linee Guida per la Valutazione e Riduzione del Rischio Sismico del Patrimonio Culturale Allineate alle Nuove Norme Tecniche per le Costruzioni (D.M. 14 Gennaio 2008). http://www.beniculturali.it/mibac/multimedia/MiBAC/documents/ 1295444865088_LINEE.pdf. Accessed on: 15 Feb. 2021.

[3] Massafra, A., Prati, D., Predari, G. \& Gulli, R., Wooden truss analysis, preservation strategies, and digital documentation through parametric 3D modelling and HBIM workflow. Sustainability, 12, p. 4975, 2020. DOI: 10.3390/su12124975.

[4] Prati, D., Zuppella, G., Mochi, G., Guardigli, L. \& Gulli, R., Wooden trusses reconstruction and analysis through parametric 3D modelling. International Archives of the Photogrammetry, Remote Sensing and Spatial Information Sciences, XLII-2/W9, pp. 623-629, 2019. DOI: 10.5194/isprs-archives-XLII-2-W9-623-201.

[5] Capra, A., Bertacchini, E., Castagnetti, C., Dubbini, M., Rivola, R. \& Toschi, I., Rilievi laser scanner per l'analisi geometrica delle torri degli Asinelli e Garisenda. INARCOS, LXVI(4), pp. 35-42, 2011.

[6] Bertacchini, E., Boni, E., Capra, A., Castagnetti, C. \& Dubbini, M., Terrestrial laser scanner for surveying and monitoring middle age tower. XXIV FIG International Congress 2010, Sydney, Australia, TS4D-n. 4445, pp. 1-13, 2010.

[7] Lusini, V., San Domenico in Camporegio. Bullettino Senese di Storia Patria, volume tredicesimo, Tip. lit. dei sordomuti di L. Lazzeri: Siena, 1906.

[8] Riedl, P.A. \& Seidl, M., Die Kirchen von Siena, 2: Oratorio della Carità-S. Domenico, Brückmann Verlag: Munchen; [poi] Polistampa: Firenze, 1985.

[9] Romagnoli, E., Biografia cronologica de' bellartisti senesi 1200-1800. Firenze SPES, 1976. 